Journal of Computer Science and Cybernetics, V.27, N.1 (2011),72-82

\title{
A FINITE VOLUME MODEL ON UNSTRUCTURED MESHES AND APPLICATION IN COMPUTING TWO DIMENSIONAL FLOWS*
}

\author{
TRAN GIA LICH ${ }^{1}, \mathrm{LE} \mathrm{DUC}^{2}$ \\ ${ }^{1}$ Institute of Mathematics, Vietnam Academy of Science and technology \\ ${ }^{2}$ National Center for Hydro-Meteorological Forecasting Hanoi, Vietnam
}

\begin{abstract}
Some methods in approximation of fluxes between adjacent cells have been proposed in context of the finite volume technique on unstructured meshes. A shallow water model has been developed for testing the proposed methods. The third order Adams-Bashforth scheme is used in integrating the governing equations. A filter is designed to remove spurious waves. The model is tested on unstructured triangular meshes with some examples in literature.

Tóm tắt. Bài báo trình bày một số kỹ thuật xấp xỉ thông lượng giữa các nốt trên lưới phi cấu trúc trong kỹ thuật thể tích hữu hạn. Các phương pháp này được thử nghiệm vào một mô hình nước nông hai chiều trên lưới tam giác phi cấu trúc. Mô hình này sử dụng sơ đồ tích phân thời gian Adams-Bashforth bậc ba. Một bộ lọc cũng được đưa vào mô hình nhằm loại bỏ các sóng nhiễu sinh ra trong quá trình tích phân. Các bài toán mẫu sẽ được sử dụng nhằm kiểm tra kỹ năng mô phỏng của mô hình.
\end{abstract}

\section{INTRODUCTION}

A model simulating flow in rivers, coastal areas,... should be able to resolve many natural phenomena in such domains. Because natural phenomena range from small scales to large scales, meshes used in models must vary and depend on problem geometries. To resolve varying geometries in complex domains, unstructured meshes are more favorable than structured uniform meshes. Finite volumes and finite elements are two numerical methods used for this kind of meshes. A cell, which is a triangular in the most simple shape, is a base element in such methods. Between these two methods, the finite volume method is more preferable than the finite element method because its flux form of equations implies the conservation of momentum and mass in the results.

The unstructured finite volume method in literature uses two techniques: Roes Approximate Riemann Solvers and Greens Theorem [4]. The key concept here is how to specify fluxes between two cells. The well-known spurious oscillations which come from a non-staggered

\footnotetext{
${ }^{*}$ This paper is supported by the Vietnam National Foundation for Science and Technology Development
} 
mesh also pose a difficulty for this method. Two techniques are given overcome those problems with complicated equations.

In this paper, we apply Greens Theorem technique and propose some simple methods in computing fluxes between adjacent cells. A two-dimensional finite volume model has been developed for testing. The model is based on shallow water equations. The third-order AdamsBashforth scheme will be used for model integration. For the first and second integration step, first-order and second-order Adams-Bashforth schemes will be used respectively. We will examine the performance of simple methods comparing with complicated methods in some test cases. A filter is also applied to eliminate spurious oscillations. This is a new feature in unstructured finite volume models.

\section{FORMULATION OF THE NUMERICAL MODEL}

\subsection{Fundamental equations}

The numerical model is based on the two dimensional Saint Venant equations in the following flux form

$$
\begin{gathered}
\frac{\partial p}{\partial t}+\frac{\partial}{\partial x}\left(\frac{p^{2}}{h}\right)+\frac{\partial}{\partial y}\left(\frac{p q}{h}\right)+\frac{\partial}{\partial x}\left(\frac{g h^{2}}{2}\right)+g h \frac{\partial z}{\partial x}-f q+\frac{\tau_{b x}}{\rho}-\frac{\tau_{w x}}{\rho}=0 \\
\frac{\partial q}{\partial t}+\frac{\partial}{\partial x}\left(\frac{p q}{h}\right)+\frac{\partial}{\partial y}\left(\frac{q^{2}}{h}\right)+\frac{\partial}{\partial y}\left(\frac{g h^{2}}{2}\right)+g h \frac{\partial z}{\partial y}+f q+\frac{\tau_{b y}}{\rho}-\frac{\tau_{w y}}{\rho}=0 \\
\frac{\partial h}{\partial t}+\frac{\partial p}{\partial x}+\frac{\partial q}{\partial y}=0
\end{gathered}
$$

where,

$p, q$ : the volume flux ( $p=u h, q=v h$ where $u, v$ are the depth-averaged current velocities);

$h$ : the instantaneous water depth;

$z$ : the bed elevation;

$g$ : the gravity acceleration;

$f$ : the Coriolis parameter;

$\rho$ : the water density;

$\tau_{b x}, \tau_{b y}:$ the bed stresses;

$\tau_{w x}, \tau_{w y}:$ the wind stresses;

For the sake of simplicity, we neglect the diffusion terms in the equations (2.1).

The equations (2.1) can be writen in a matrix system of the form

$$
\frac{\partial}{\partial t}\left(\begin{array}{c}
p \\
q \\
h
\end{array}\right)+\frac{\partial}{\partial x}\left(\begin{array}{c}
\frac{p^{2}}{h}+\frac{g h^{2}}{2} \\
\frac{p q}{h} \\
p
\end{array}\right)+\frac{\partial}{\partial y}\left(\begin{array}{c}
\frac{p q}{h} \\
\frac{q^{2}}{h}+\frac{g h^{2}}{2} \\
q
\end{array}\right)=\left(\begin{array}{c}
-g h \frac{\partial z}{\partial x}+f q-\frac{\partial \tau_{b x}}{\partial \rho}+\frac{\partial \tau_{w x}}{\partial \rho} \\
-g h \frac{\partial z}{\partial y}-f q-\frac{\partial \tau_{b y}}{\partial \rho}+\frac{\partial \tau_{w y}}{\partial \rho} \\
0
\end{array}\right) .
$$


In a short form, we can write

$$
\frac{\partial}{\partial t}(V)+\frac{\partial}{\partial x}(E)+\frac{\partial}{\partial y}(F)=G,
$$

and the vectors $V, E, F$, and $G$ can trace back to the equation (2.2a).

\subsection{Numerical methods}

Now to apply the equation $(2.2 \mathrm{~b})$ in context of the finite volume method, we integrate it over an area $A$ whose bounding curve is $C$.

$$
\iint_{A} \frac{\partial V}{\partial t} d S+\iint_{A} \frac{\partial E}{\partial x} d S+\iint_{A} \frac{\partial F}{\partial y} d S=\iint_{A} G d S .
$$

With the help from Greens theorem, two surface integrals for fluxes $E$ and $F$ define two line integrals

$$
\iint_{A} \frac{\partial V}{\partial t} d S+\oint_{C} E d y-\oint_{C} F d x=\iint_{A} G d S .
$$

Considering two surface integrals in (2.4) as definition for mean values, we have

$$
\frac{\partial V}{\partial t} A+\oint_{C} E d y-\oint_{C} F d x=\bar{G} A .
$$

The equation (2.5) shows that the local change of area-averaged momentum and mass in the area $A$ depend on the source-sink term $G$ and fluxes go into $A$ through $C$. Like other finite volume models, $A$ has a form of polygon which consists of a number of lines $I$, each denoted by $L_{i}(i=1, I)$. For consistence with finite volume terms, we will call the area $A$ as a cell or node $A$ with its vertices and edges. Then, the final form of governing equation in our model is

$$
\frac{\partial \bar{V}}{\partial t}=-\frac{1}{A} \sum_{i=1}^{I} \int_{L_{i}} E d y+\frac{1}{A} \sum_{i=1}^{I} \int_{L_{i}} F d x+\bar{G} .
$$

Some terms in $G$ have nonlinear forms like bottom frictions or topographic gradients, and evaluating these terms needs a linear procedure, but it should not be a problem. The main difficulty involves in computing the fluxes through each edge $L_{i}$. We need an approximation for the integral

$$
\int_{L_{i}} F d x=\int_{x_{i}}^{x_{i+1}} F d x
$$

where we only demonstrate the integral with $F$ fluxes. The integral with $E$ fluxes is treated similar. The simplest approximation to the integral is the midpoint rule 


$$
\int_{x_{i}}^{x_{i+1}} F d x=\bar{F} \Delta x_{i} \approx F_{i+1 / 2} \Delta x_{i} .
$$

Here the integral is approximated as a product of the flux $F$ at the line center and the difference $\Delta x_{i}$. There are other approximation methods like the trapezoid rule or Simpsons rule in [6], in this paper we only use the midpoint rule. Because the values of $\mathrm{F}$ in the line are not available and only the area-averaged values are calculated so an approximation must be introduced.

To approximate the value of $F_{i+1 / 2}$, we will compute the flux at the left and right of the midpoint of line $L_{i}$. In the Figure 2.1, two cells are shown together with the notations we shall use. Two adjacent cells $M_{i}$ and $M_{j}$ have the same edge AB with the midpoint $\mathrm{O}$. The cells are in triangular forms but the method demonstrated here is the same for any forms, even adjacent cells have different forms. The distance between the center of the left cell $M_{i}$ and the midpoint $\mathrm{O}$ is denoted by a vector $r_{i}$ and the center of the right cell $M_{j}$ and the midpoint $\mathrm{O} r_{j}$. The value of $F_{O}$ can be computed through the left flux $F_{l}$ and the right flux $F_{r}$ at the midpoint $\mathrm{O}$.

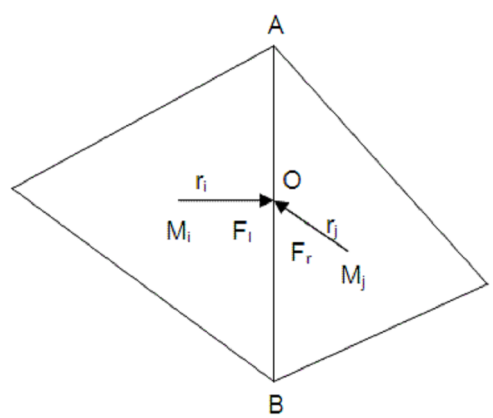

Figure 2.1. Two adjacent cells and the notation used for approximating fluxes between two cells

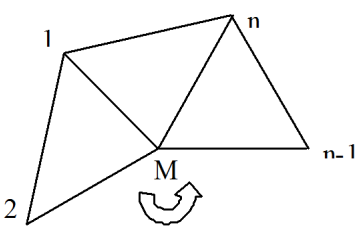

Figure 2.2. The cell center $\mathrm{M}$ with other surrounding points in formulating partial derivative approximations

The upwind scheme will be used in this case. In Figure 2.2, with the same notation in the Figure 2.1, we show the flow velocity $v$ between two cells in addition. The velocity vector has an angle $\alpha$ with respect to the line connecting two cell centers. Then the value of $F$ at the midpoint can be computed with a method equivalent to the "well-known" upwind method

$$
F_{O}=\frac{(1+\cos \alpha)}{2} F_{1}+\frac{(1-\cos \alpha)}{2} F_{r}
$$


If $\alpha=0$, this formulation reduce to the strict upwind formulation $F_{O}=F_{l}$. To get the value of $\alpha$, we must know the mean velocity vector $v$ between two cells. This vector will be approximated by two area-averaged velocity vector $v_{i}$ and $v_{j}$ from two cells using the cell area weighted mean

$$
\nu=\frac{S_{i} \nu_{i}+S_{j} \nu_{j}}{S_{i}+S_{j}}
$$

The remain problem is how to compute the value of Fl and Fr. A second-order approximation is available if we know the value of $\mathrm{F}$ at the cell center and its directional derivative, which estimates the left and right fluxes as

$$
F_{l}=F_{i}+\left(\frac{\partial F}{\partial r}\right)_{i} r_{i}, F_{r}=F_{j}+\left(\frac{\partial F}{\partial r}\right)_{j} r_{j} .
$$

The values of $F_{i}$ and $F_{j}$ can be approximated by the area-averaged values of $F$ at the cell centers. The directional derivatives are decomposed in two components with respect to $\mathrm{Ox}$ and $\mathrm{Oy}$ axes as

$$
\left(\frac{\partial F}{\partial r}\right)_{i} r_{i}=\left(\frac{\partial F}{\partial x}\right)_{i} r_{x i}+\left(\frac{\partial F}{\partial y}\right)_{i} r_{y i},\left(\frac{\partial F}{\partial r}\right)_{j} r_{j}=\left(\frac{\partial F}{\partial x}\right)_{j} r_{x j}+\left(\frac{\partial F}{\partial y}\right)_{j} r_{y j}
$$

All the partial derivatives are obtained by interpolation of the vertex values or the nodal values. This interpolation method is illustrated in Figure 2.3, $\mathrm{M}$ is a cell center, where we want to approximate the spatial derivatives. We will numerate the points which link with $\mathrm{M}$ in an anti-clockwise sequence $1,2, \ldots, n$ (Figure 2.3). These points can be considered as the cell vertices or other cell centers which are neighbors of $\mathrm{M}$. The area of the polygon by the edges $12,23, . ., n 1$ is denoted by $\mathrm{S}$ with the bounding curve $\mathrm{C}$.

Applying the Greens theorem for spatial derivatives with an integration over S, we have

$$
\iint_{S} \frac{\partial F}{\partial x} d S=\oint_{C} f d y, \iint_{S} \frac{\partial F}{\partial y} d S=-\oint_{C} f d x
$$

Now assuming a piecewise constant approximation to the spatial derivatives inside the polygon $\mathrm{S}$, we have

$$
\frac{\partial F}{\partial x} \approx \frac{\partial \bar{F}}{\partial x}=\frac{1}{S} \oint_{C} f d y, \frac{\partial F}{\partial x} \approx \frac{\partial \bar{F}}{\partial x}=-\frac{1}{S} \oint_{C} f d x .
$$

Two integrals over the closed $C$ in the equation (2.19) are the sum of the integrals over the edges $12,23, \ldots, n 1$. With a simple linear approximation on each integral, we obtain

$$
\begin{aligned}
\oint_{C} F d y & =\int_{12} F d y+\int_{23} F d y+\ldots+\int_{n 1} F d y \\
& =\frac{F_{1}+F_{2}}{2}\left(y_{2}-y_{1}\right)+\frac{F_{2}+F_{3}}{2}\left(y_{3}-y_{2}\right)+\ldots+\frac{F_{n}+F_{1}}{2}\left(y_{1}-y_{n}\right) .
\end{aligned}
$$




$$
\begin{aligned}
\oint_{C} F d x & =\int_{12} F d x+\int_{23} F d x+\ldots+\int_{n 1} F d x \\
& =\frac{F_{1}+F_{2}}{2}\left(x_{2}-x_{1}\right)+\frac{F_{2}+F_{3}}{2}\left(x_{3}-x_{2}\right)+\ldots+\frac{F_{n}+F_{1}}{2}\left(x_{1}-x_{n}\right) .
\end{aligned}
$$

If the points $1, \ldots, n$ in the Figure 2.3 are the surrounding cell centers of $M$, the value of $F_{i}$ is the area-averaged value of $F$ corresponding to the cell $i$.

If these points are the cell vertices, the value of $F_{i}$ is obtained by the cell area weighted interpolation methods (Figure 2.4)

$$
F_{A}=\frac{\overline{F_{1}} S_{1}+\ldots+\overline{F_{n}} S_{n}}{S_{1}+\ldots+S_{n}}
$$

where $S_{i}$ is the area of cell $i$, which has the point A as its vertex and $F_{i}$ is the value of $F$ averaged over this cell.

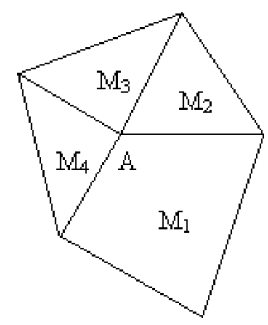

Figure 2.4. Neighbor cells with the same vertex A and the notation used for interpolation method of any field at A

To integrate the equation (2.6), we use the third-order Adams-Bashforth scheme because of its highly accurate (third order in time) and economical (explicit method) property.

$$
V^{n+1}=V^{n}+\frac{1}{12}\left(23 \frac{\partial V^{n}}{\partial t}-16 \frac{\partial V^{n-1}}{\partial t}+5 \frac{\partial V^{n-2}}{\partial t}\right) \Delta t .
$$

The first-order and second-order Adams-Bashforth schemes are applied in the first and second integration step, respectively.

In testing the model with shock waves or supercritical flows, high frequency oscillations occur in the solutions, amplify very fast and overcome all slow waves. To filter out such waves from solutions a filter is applied. The filter will be carried out a given time for all points after a given step. For a field like $h$, after each filter step its value at a point $M$ is

$$
h_{M}=(1-w) \times h_{M}+w \times \bar{h}_{M},
$$

where $w$ is the filter weight (0.02 in this model) and the average of $h$ is computed from the surrounding cells by arithmetic mean or weighted mean with respect to cell areas or cell 
distances. With the filter (2.18) the conservation of mass may be violated but we found that it is not significant in practice as shown in the next section.

\subsection{Boundary conditions}

Without the diffusion term, the equations (2.1) can be transformed into the symmetric form which is quasi-linear hyperbolic. The eigenvalues of the flux Jacobian matrix are phase speeds of waves travelling in or out the domain. The wave speeds depend on the normal velocity $u_{n}$ and the gravity wave velocity $c$. The number of boundary conditions equal to the number of waves travelling in the domain. So the number of boundary conditions is problem-oriented and we need a general frame in implementing boundary conditions.

Suppose that $L$ is an edge of a cell $M$, which coincides with the domain boundary and the right side of $L$ belongs to the cell while the left side of $L$ is out of domain. We can compute the right flux $F_{r}$ with any method demonstrated in the preceding section but it is not the case with the left flux $F_{l}$. For this value, we need some special procedures depending on boundary conditions. Here are the methods supported in the model:

- If not specified, the left fluxes are equal to the right fluxes.

- For closed boundary conditions, the tangential components of left fluxes $p, q$ are set to ones of right fluxes. The normal components of both right and left fluxes are zero.

- For imposed boundary conditions (discharge $p, q$ or water depth $h$ ), the left fluxes are set to the imposed value.

\section{MODEL TESTING}

In the following section, some tests are carried out to validate the model performance. To generate unstructured meshes, we use a free software named TRIANGLE. This program enables creating unstructured triangular meshes for any complicated geometry. That means all tests are implemented on unstructured triangular meshes although the model is developed for any kind of unstructured meshes.

In the following figures, the analytical solution (optional) will be shown by a solid line while the numerical solution, because of the nature of unstructured meshes, could only plot as a point for each cell. This is only true for one-dimensional tests. With two dimensional tests, vector fields or height fields will be shown with an appropriate interpolation method.

\subsection{Dam break over a wet or dry bed}

The problem configuration is shown in Figure 3.1. There is a dam between two water layers with the depths $h_{r}$ and $h_{l}$ respectively. The dam is supposed to vanish instantaneously.

In testing we set $h_{l}=6 \mathrm{~m}, h_{r}=2 \mathrm{~m}$ for the wet bed test and $h_{r}=0.001 \mathrm{~m}$ for the dry bed test. The channel length is $1000 \mathrm{~m}$, the channel width is $4 \mathrm{~m}$ and the average distance between two adjacent cells is $2 \mathrm{~m}$. The closed boundary conditions are imposed in left and 
right boundaries. The analytical solutions compared to the numerical solutions after $30 \mathrm{~s}$ in both cases are shown in Figure 3.2. The time step is $0.1 \mathrm{~s}$ and the filter number is 5 for each time step.

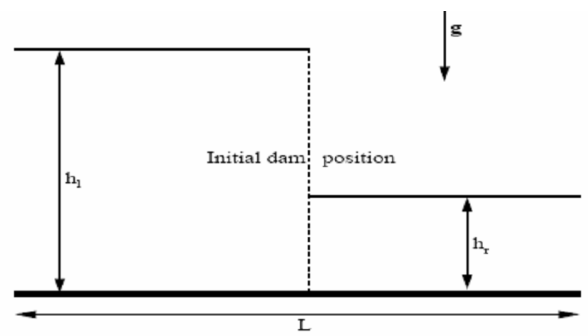

Figure 3.1. The geometry of dam break problem over a wet bed
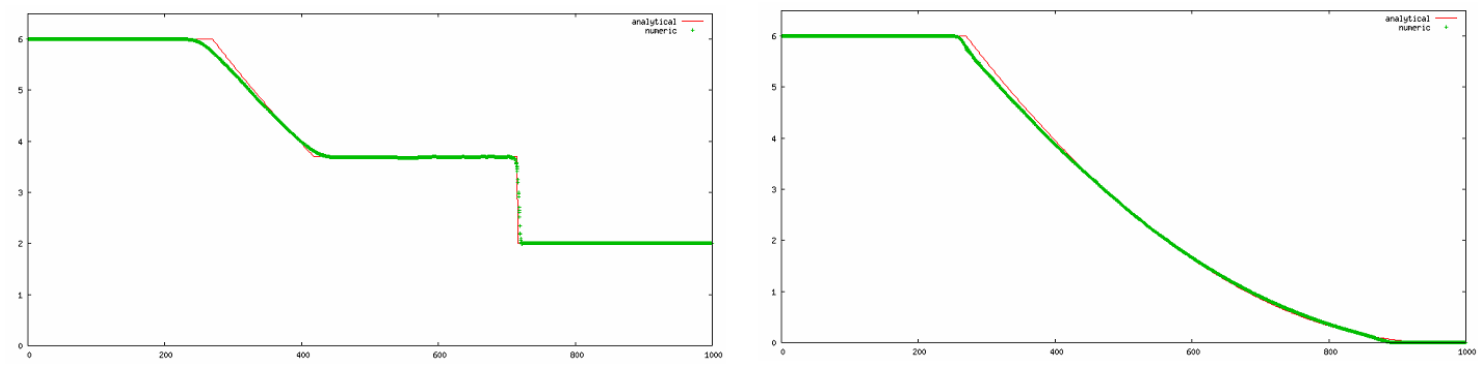

Figure 3.2. The numerical solutions compared to the analytical solutions after 30s for dam break problem over a wet bed (left) and a dry bed (right)

\subsection{Partial dam break}
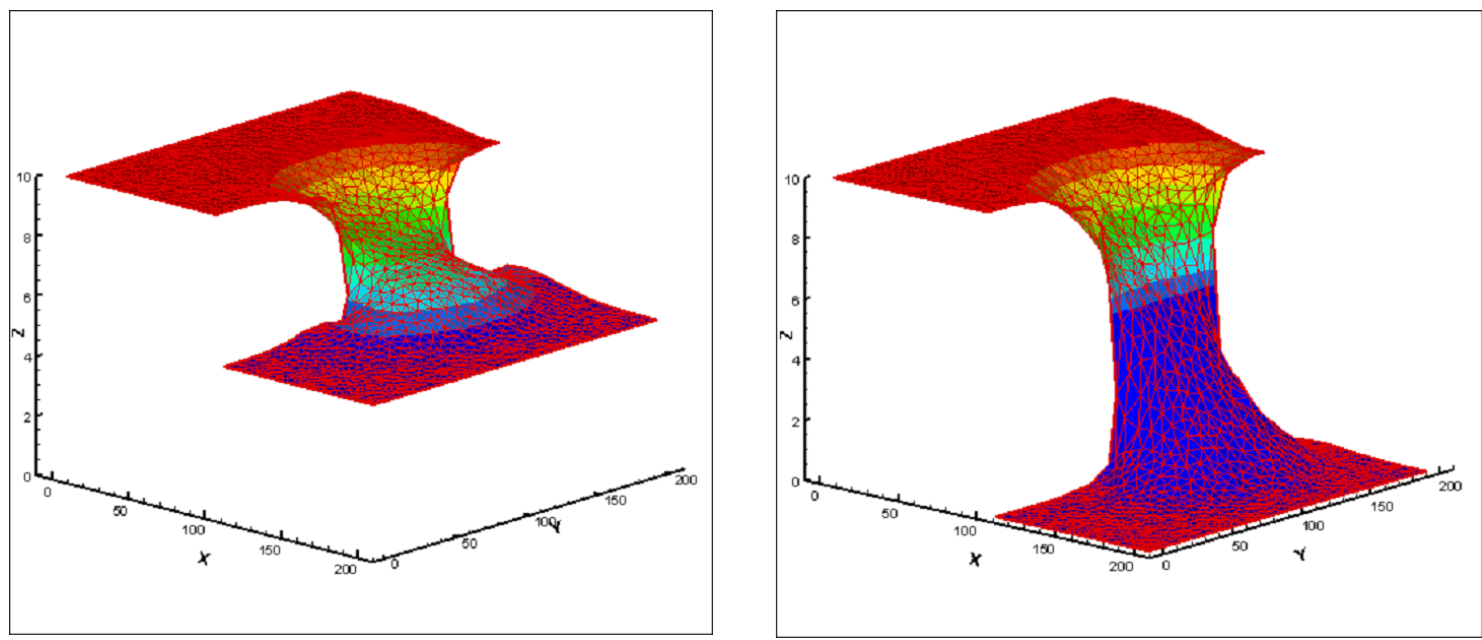

Figure 3.3. The numerical results integrated after $5 \mathrm{~s}$ for partial dam break problem over a flat bed with the downstream initial water depth $5 \mathrm{~m}$ (left) and $0.001 \mathrm{~m}$ (right)

This problem is a general case in two dimensional space of the preceding problem. It is proposed by Fennema [5]. All initial conditions are the same as in the one dimensional 
case where a dam dividing the domain into two water layers with different depths. There is a breach separating the dam into two asymmetric parts. The breach is assumed occurring instantaneously. In his paper Fennema set $h_{l}=10 \mathrm{~m}, h_{r}=5 \mathrm{~m}$ and the domain consisting of a $200 \mathrm{~m} \times 200 \mathrm{~m}$ region which is subdivided into a $41 \times 41$ square grid. The breach is $75 \mathrm{~m}$ wide and centered at $75 \mathrm{~m}$. However, Alcrudo [2] argued that the downstream water depth $5 \mathrm{~m}$ is not a severe test for the model because the flow is subcritical everywhere. Therefore, the much smaller downstream water depth should be tested for the numerical scheme. Test results after $5 \mathrm{~s}$ with two downstream water depth $5 \mathrm{~m}, 0.001 \mathrm{~m}$ are shown in the Figure 3.3. The problem configuration is consistent with Fennema. All boundaries are solid. The time step is 0.1s and the filter number is 5 for each time step.

These results are very similar to others found in the literature for this problem (Anastasiou $[3])$.

\subsection{Circular dam break}

This is another problem in the test set involving dam break problems. The problem was suggested by Alcrudo [1]. The initial conditions consist of two states separated by a circular discontinuity. The radius of the circle $r=11 \mathrm{~m}$ and it is centered at $x=y=25 \mathrm{~m}$. Both components of the initial fluxes $p$ and $q$ are set to zero everywhere and $h$ is set to $10 \mathrm{~m}$ within the circle and $1 \mathrm{~m}$ outside the circle. In testing, we also model the flows when the domain outside the circle is dry. The domain is a square $50 \mathrm{~m} \times 50 \mathrm{~m}$ with the distance between two points is $1 \mathrm{~m}$. The solutions are sought when $t=0.69 \mathrm{~s}$ (Figures 3.4 ). The time step is $0.01 \mathrm{~s}$ and the filter number is 4 for each time step.
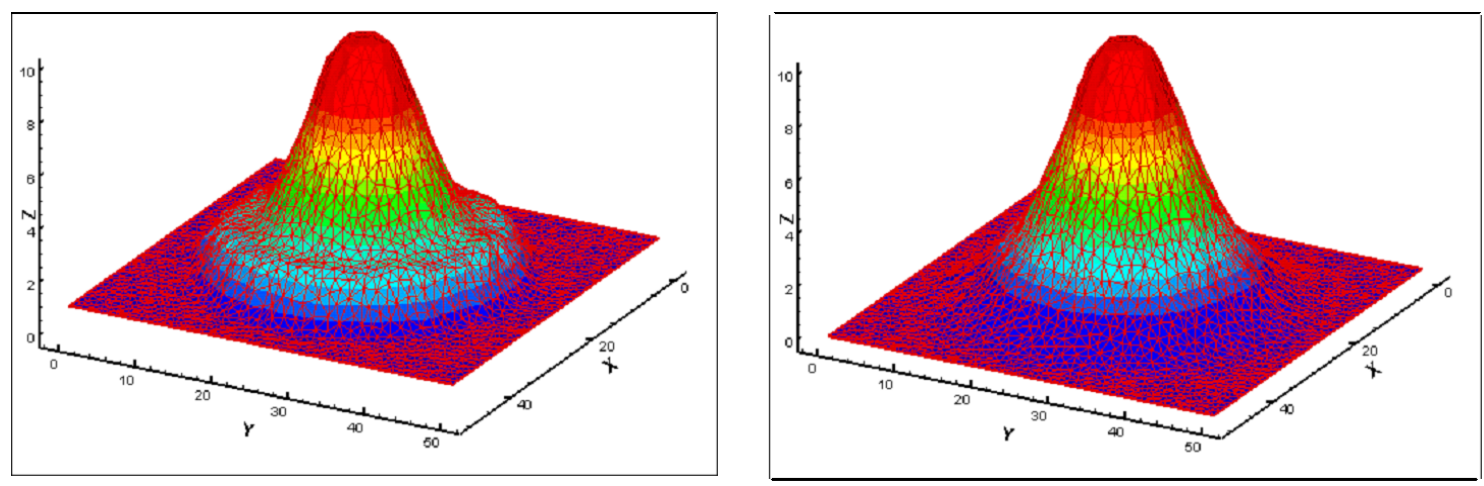

Figure 3.4. The numerical results integrated after $0.69 \mathrm{~s}$ for circular dam break problem over a flat bed with the initial water depth $1 \mathrm{~m}$ (left) and $0.001 \mathrm{~m}$ (right)

Similar results were obtained by Alcrudo [1] and Anastasiou [3]. 


\subsection{Reflection of surge waves}

This test is adopted from $\mathrm{Hu}$ [7]. The geometry is a rectangular channel $10 \mathrm{~km}$ long and $50 \mathrm{~m}$ width. One end of the channel is closed with a solid wall. The other end of the channel is an inlet boundary where a surge with water depth $d_{1}=10 \mathrm{~m}$ is imposed. Initially, water level is at rest $d_{2}=5 \mathrm{~m}$. The surge wave travels from left to right. When the wave hits the right solid wall, a reflected surge wave with increased depth of $d_{3}$ travels in the opposite direction. The numerical results compared to the analytical results are shown in the Figure 3.5. The spatial step is $25 \mathrm{~m}$, the time step is $1 \mathrm{~s}$.
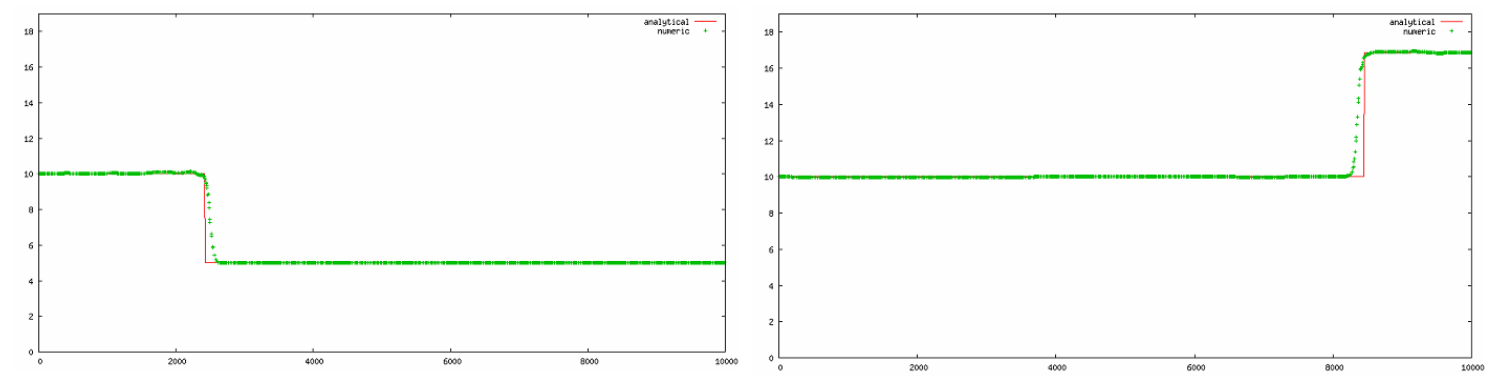

Figure 3.5. The numerical solutions compared to the analytical solutions after 200s (left) and 1000s (right) for surge wave problem over a flat bed

\subsection{Flow over obstacles}

A vortex after a sluice in a channel: Along a rectangular channel $L=10 \mathrm{~m}$ with a constant width $B=2 \mathrm{~m}$, there is a sluice with length $2 \mathrm{~m}$ making an angle $30^{\circ}$ with the channel bank and located $4 \mathrm{~m}$ from the upstream end. Two water depths are imposed at two ends of channel: $2.5 \mathrm{~m}$ and $1.9 \mathrm{~m}$. After the sluice, a vortex occurs (Figure 3.6).

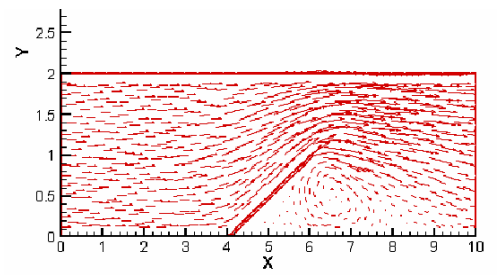

Figure 3.6. The numerical solution for flow in a channel with a $30^{0}$ sluice

\section{CONCLUSION}

All numerical results in testing show a similarity with many results in literature. This leads to an impress about a good performance of the model. From 12 ways of approximation for fluxes between two adjacent cells, the upwind scheme combined with the second-order interpolation method is shown as the best candidate. Because the filter smoothes out the results, there are not any significant differences among other combinations. However, more 
severe tests from other problems are needed to validate the model, especially in computing realistic flows.

Spurious waves occur in the results, which come from unstructured meshes and the approximation techniques. The filter step is also necessary in modeling. Then we confront a problem concerning the conservation of mass. This problem is not severe in the tests but we need examine it in future. The most difficult thing lies in choosing an appropriate number of filter times. Large filter numbers will smooth the solutions drastically, while small filter numbers support spurious waves and the solutions will be distorted. We can say about a sensitivity of solutions on number of filter times.

\section{REFERENCES}

[1] F.Alcrudo F, PG. Navarro, A high-resolution Godunov-type scheme in finite volumes for the 2D shallow water equations, International Journal for Numerical Methods in Fluids 16 (1993) 489-505.

[2] F. Alcrudo, PG. Navarro, Computing two dimensional flood propagation with a high resolution extension of McCormack's method, Proceedings on modeling of flood propagation over initially dry areas, American Society of Civil Engineers, Milan, Italy, 1994 (3-17).

[3] K. Anastasiou, CT. Chan, Solution of the 2D shallow water equations using the finite volume method on unstructured triangular meshes, International Journal for Numerical Methods in Fluids 24 (1997) 1225-1245.

[4] KN. Dan, HV.Lai, and Y. Xinya, 2-D shallow-water models using unstructured finitevolumes methods, Caen 2002

[5] RJ. Fennema, MH. Chaudhry, Explicit methods for 2D transient free surface flows, American Society of Civil Engineers, Journal of Hydraulic Engineering 116 (1990) 1013-1034.

[6] JH. Ferziger, M. Peric, Computational methods for fluid dynamics, Springer Verlag, 2002 (pp.423).

[7] K. Hu, CG. Mingham, DM. Causon, Numerical simulation of wave overtopping of coastal structures using the non-linear shallow water equations, Coastal Engineering 41 (2000) $433-465$.

Received on April 14 - 2009

Revised on October 26 - 2010 